\title{
Numerical simulation of wave propagation in magnetic network
}

\author{
G. Vigeesh ${ }^{1}$, S. S. Hasan ${ }^{1}$ and O. Steiner ${ }^{2}$ \\ ${ }^{1}$ Indian Institute of Astrophysics, \\ Bangalore-560034, India \\ email: vigeesh@iiap.res.in, hasan@iiap.res.in \\ ${ }^{2}$ Kiepenheuer-Institut für Sonnenphysik, \\ 79104 Freiburg, Germany \\ email: steiner@kis.uni-freiburg.de
}

\begin{abstract}
We present 2-D numerical simulations of wave propagation in the magnetic network. The network is modelled as consisting of individual magnetic flux sheets located in intergranular lanes. They have a typical horizontal size of about $150 \mathrm{~km}$ at the base of the photosphere and expand upward and become uniform. We consider flux sheets of different field strengths. Waves are excited by means of transverse motions at the lower boundary, to simulate the effect of granular buffeting. We look at the magneto-acoustic waves generated within the flux sheet and the acoustic waves generated in the ambient medium due to the excitation. We calculate the wave energy fluxes separating them into contributions from the acoustic and the Poynting part and study the effect of the different field strengths.
\end{abstract}

Keywords. Sun: magnetic fields - magnetohydrodynamics (MHD) - Sun: photosphere oscillations

The magnetic network in the solar atmosphere consists of intense magnetic field elements located in intergranular lanes. These magnetic elements have field strengths ranging from few hundred gauss to kilogauss (Berger et al. 2004). Granular buffeting is believed to excite MHD waves in this medium. Following Hasan et al. (2005) and Hasan \& Ballegooiijen (2008), we model network magnetic fields as non-potential structures having a horizontal size of about $150 \mathrm{~km}$ at the base of the photosphere. Waves are excited in them by means of transverse motions at the lower boundary. The driving generates both fast and slow waves within the flux sheet and acoustic waves in the ambient medium. We consider flux sheets of different field strengths and study the energy transported by the these waves.

The boundary conditions for the magnetic field and the pressure distribution in the physical domain is specified initially. The magnetic field configuration and the density distribution is then iteratively calculated from the magnetohydrostatic equations. We treat two different cases corresponding to field strengths (at $z=0$ ) of $800 \mathrm{G}$ (moderate field case) and $1600 \mathrm{G}$ (strong field case), on the axis of the sheet. Waves are excited in this structure by an impulsive transverse motions of the lower boundary with a period of $24 \mathrm{~s}$ and an amplitude of $750 \mathrm{~m} \mathrm{~s}^{-1}$. We solve the MHD equations following the numerical method described in Steiner et al. (1994).

The wave energy flux is the energy that is transported by the waves. The linearized wave energy flux is given as (Bray \& Loughhead 1974, Bogdan et al. 2003),

$$
\mathbf{F}_{\text {wave }}=\Delta p \mathbf{v}+\frac{1}{4 \pi}\left(\mathbf{B}_{0} \cdot \Delta \mathbf{B}\right) \mathbf{v}-\frac{1}{4 \pi}(\mathbf{v} \cdot \Delta \mathbf{B}) \mathbf{B}_{0}
$$



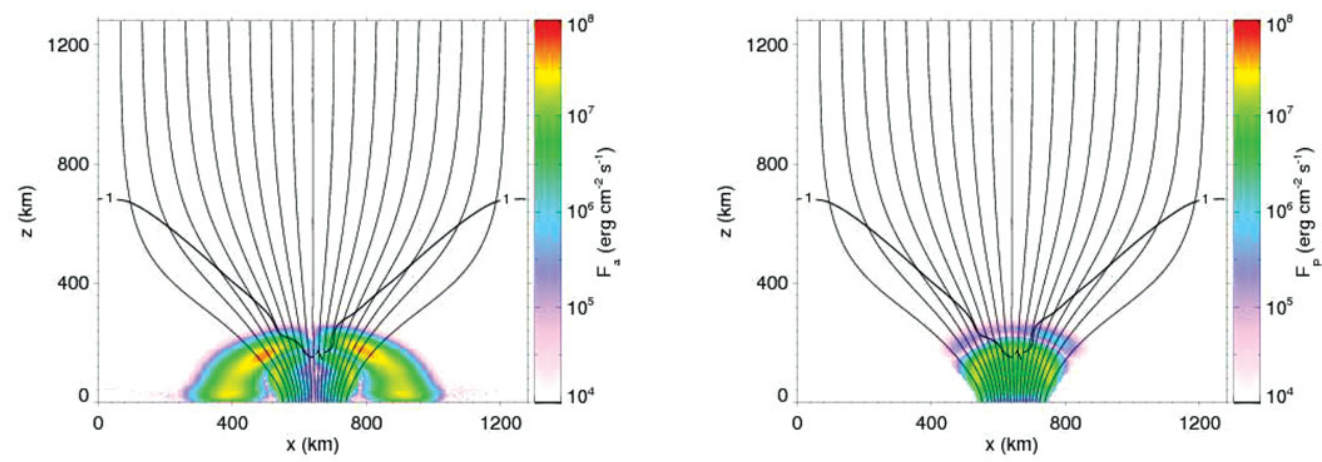

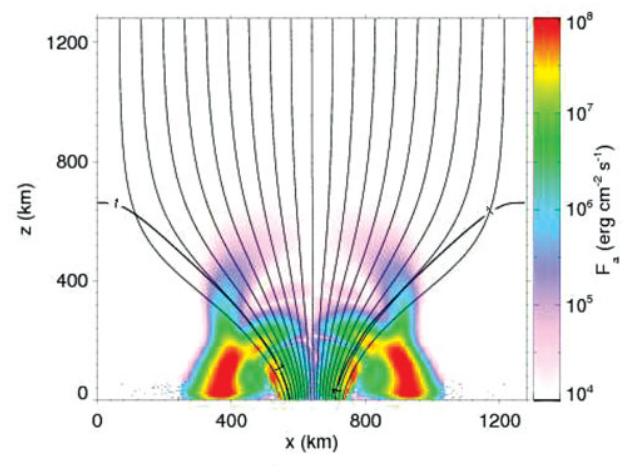

a) Acoustic

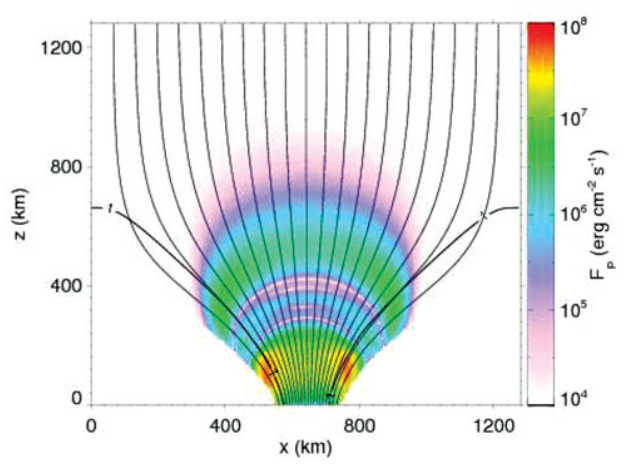

b) Poynting

Figure 1. Wave energy fluxes for the case in which the field strength at the axis at $z=0$ is $800 \mathrm{G}$ (top panels) and $1600 \mathrm{G}$ (bottom panels). The colors show (a) the acoustic flux, and (b) the Poynting flux, at $40 \mathrm{~s}$ due to an impulsive horizontal motion at the $z=0$ boundary with an amplitude of $750 \mathrm{~m} \mathrm{~s}^{-1}$ and a period of $P=24 \mathrm{~s}$. The thin black curves are the field lines and the thick black curve represents the contour of $\beta=1$. The Poynting fluxes are not shown in the ambient medium

The first term is the acoustic flux, and the last two terms are the Poynting flux. The operator $\Delta$ gives the perturbations in the variable with respect to the initial time and $B_{0}$ refers to the unperturbed magnetic field. Fig. 1. shows the magnitude of acoustic and Poynting fluxes at an elapsed time of $40 \mathrm{~s}$ for the two cases under consideration.

We see that the acoustic flux transport is isotropic, while the Poynting fluxes are localized within the flux tube. In the case of moderate field, the excitation at the bottom boundary generates fast (acoustic) and slow (magnetic) waves. These waves undergo mode transmission and conversion (Cally, 2007) as they cross the $\beta=1$ layer. On the other hand, in the case of the strong field, the fast (magnetic) and slow (acoustic) waves generated within the flux tude do not encounter the $\beta=1$ layer. There is a significant amount of acoustic emission into the ambient medium in the case of the flux tube with strong field. This is due to the sharp change in the magnetic field strength across the boundary between the flux tube and ambient medium.

To summarize our results, we find that the magnetoacoustic modes generated in the two field configurations are different. The acoustic wave emission from the tube interface depends on the strength of the field. We see a stronger emission in the case of a flux tube with strong field. We conclude that the magnetic field strength significantly effect the wave generation and propagation in the magnetic atmosphere. 


\section{Acknowledgements}

This work was supported by the German Academic Exchange Service (DAAD), grant D/05/57687, and the Indian Department of Science \& Technology (DST), grant DST/ INT/DAAD/P146/2006.

\section{References}

Berger, T. E., et al. 2004, A\&A 428, 613

Bogdan, T. J. et al. 2003, ApJ 599, 626

Bray, R. J. \& Loughhead, R. E. 1974, The Solar Chrmosphere (London: Chapman \& Hall)

Cally, P. S. 2007, Astronomische Nachrichten 328, 286

Hasan, S. S., van Ballegooiijen, A. A., Kalkofen W., \& Steiner, O. 2005, ApJ. 631, 1270

Hasan, S. S. \& van Ballegooiijen, A. A. 2008, ApJ 680, 1542

Steiner, O., Knölker M., \& Schüssler, M. 1994, NATO ASI Ser. C-433, (Dordrecht: Kluwer), p. 441 